\title{
Conhecimento e prática de enfermeiros no controle de feridas neoplásicas
}

\author{
Nurses' knowledge and practice in the neoplastic wounds \\ control
}

\author{
Glenda Agra ${ }^{1}$ • Maria Vitória de Souza Medeiros ${ }^{2}$ Débora Thaise Freires de Brito ${ }^{3}$ Fábia Letícia Martins de Andrade ${ }^{4}$ \\ Edlene Régis Silva Pimentel ${ }^{5} \cdot$ Marta Miriam Lopes Costa $^{6}$
}

\begin{abstract}
RESUMO
O objetivo deste estudo foi verificar o conhecimento e prática de enfermeiros no controle de sintomas de pacientes com feridas neoplásicas. Trata-se de um estudo descritivo de desenho quantitativo realizado com 22 enfermeiros de um hospital filantrópico de Campina Grande - PB durante o período de abril a junho de 2016. 0 instrumento foi um questionário estruturado, contendo questões relativas ao controle do sangramento, exsudato, odor, necrose e fístula das feridas neoplásicas. Os resultados obtidos nesta pesquisa permitiram identificar que os enfermeiros apresentam lacunas no conhecimento de conteúdos e técnicas sobre avaliação e tratamento de pacientes com feridas neoplásicas. Além disso, constatou-se que os enfermeiros não executam alguns cuidados pertinentes a essa clientela. Desse modo, conclui-se que a instituição precisa investir em educação permanente, a fim de treinar a equipe de enfermagem para o acompanhamento de pacientes com lesões neoplásicas, adquirir materiais necessários e implantar protocolos assistenciais que norteiem a prática de métodos avaliativos e terapêuticos para o cuidado com pessoas com feridas neoplásicas, familiares e cuidadores.
\end{abstract}

Palavras-chave: Conhecimento; Enfermeiros; Cuidados de enfermagem; Assistência ao Paciente; Neoplasias cutâneas.

\begin{abstract}
The aim of this study is to verify the nurses' knowledge and practice in the control of symptoms of patients with neoplastic wounds. This is a descriptive study of quantitative design performed with 22 nurses from a philanthropic hospital in Campina Grande - PB, during the period from April to June 2016. The instrument was a structured questionnaire, containing questions related to the control of bleeding, exudate, odor, necrosis and fistula of neoplastic wounds. The results obtained in this research allowed to identify that the nurses have gaps in the knowledge of contents and techniques on the evaluation and treatment of patients with neoplastic wounds. In addition, it was found that the nurses do not perform some pertinent care to this clientele. Thus, it concluded that the institution needs to invest in permanent education, in order to train the nursing team to follow up patients with neoplastic lesions, acquire necessary materials and implement care protocols that guide the practice of evaluative and therapeutic methods for the care of people with neoplastic wounds, relatives and caregivers.
\end{abstract}

Keywords: Knowledge; Nurses; Nursing Care; Patient Care; Cutaneous Neoplasms.

${ }^{1}$ Psicóloga clínica. Especialista em Psicologia Hospitalar. Enfermeira. Especialista em Terapia Intensiva e em Cuidados Paliativos. Mestre na Atenção à Saúde do Adulto. Doutoranda do Programa de Pós-Graduação em Enfermagem da Universidade Federal da Paraíba UFPB). Docente da Universidade Federal de Campina Grande (UFCG). Membro Adjunto da Academia Nacional de Cuidados Paliativos. Membro do Grupo de Estudos e Pesquisas em Tratamento de Feridas da Universidade Federal da Paraíba (GEPEFE/UFPB).João Pessoa-Paraíba,Brasil. Email: glendaagra@ outlook.com. Autor correspondente.

${ }^{2}$ Enfermeira. Universidade Federal de Campina Grande (UFCG-PB). Campina Grande, Paraíba, Brasil. Email: vitoria junco@yahoo.com.br.

${ }^{3}$ Enfermeira. Residente do Programa Multiprofissional da Universidade Federal da Paraíba e Prefeitura Municipal de João Pessoa (UFPB).João Pessoa - Paraíba, Brasil. Email: deborathaise_@ hotmail.com.

${ }^{4}$ Acadêmica de Enfermagem da Universidade Federal de Campina Grande (UFCG). Campina Grande, Paraíba, Brasil. Email: lethyciaandrade@hotmail.com.

${ }^{5}$ Enfermeira. Docente da Universidade Federal de Campina Grande (UFCG). Campina Grande, Paraíba, Brasil. Email: edleneregis@hotmail.com

${ }^{6}$ Enfermeira. Docente dos Cursos de Graduação e Pós-Graduação em Enfermagem da Universidade Federal da Paraíba (UFPB). Doutora em Enfermagem e em Sociologia. Membro do Grupo de Estudos e Pesquisas em Tratamento de Feridas da Universidade Federal da Paraíba (GEPEFE/UFPB). Coordenadora Geral da Divisão Técnica de Enfermagem do Hospital Universitário Lauro Wanderley (HULW/UFPB).João Pessoa - Paraíba, Brasil. Email: marthamiryam@hotmail.com. 


\section{INTRODUÇÃO}

O Brasil vivencia um fenômeno conhecido como transição epidemiológica, cuja característica é a mudança nas causas de mortalidade e morbidade associada às transformações demográficas sociais e econômicas. Nessa conjuntura, as doenças crônicas não transmissíveis, dentre elas, o câncer, aparecem como uma das doenças responsáveis pela modificação do perfil de adoecimento da população brasileira; e apesar dos avanços em pesquisas e novas modalidades terapêuticas existentes, é considerada a segunda maior causa de mortalidade no país ${ }^{1}$.

Câncer é definido como uma doença genética caracterizada pelo crescimento e divisão celular desordenados e, por isso, chamado de tumor maligno, que, por usa vez, pode invadir tecidos e órgãos adjacentes e/ou distantes, denominados de metástases².

A estimativa de câncer para o Brasil, para o biênio 20162017, aponta a ocorrência de cerca de 600 mil casos novos; destes, 180 mil casos, aproximadamente, serão câncer de pele não melanoma. 0 perfil epidemiológico revela que os cânceres mais frequentes, exceto o de pele não melanoma, serão o câncer de próstata (61 mil) em homens e mama (58 mil) em mulheres. Já os tipos de câncer mais frequentes em homens serão próstata (28,6\%), pulmão (8,1\%), intestino (7,8\%), estômago (6,0\%) e cavidade oral $(5,2 \%)$; e em mulheres, os cânceres de mama (28,1\%), intestino (8,6\%), colo do útero (7,9\%), pulmão $(5,3 \%)$ e estômago $(3,7 \%)$ figurarão entre os principais. Embora haja limitações, acredita-se que as estimativas sejam capazes de descrever padrões atuais de incidência de câncer, possibilitando o dimensionamento da magnitude e do impacto dessa doença no Brasil. Para estes mesmos anos, na região Nordeste, estima-se que ocorram 47.520 casos novos de neoplasia em homens e 51.540 em mulheres ${ }^{3}$.

As feridas neoplásicas também denominadas lesões oncológicas, malignas, tumorais ou fungóides (quando apresentam aspecto de cogumelo ou couve-flor) são formadas pela infiltração das células malignas do tumor nas estruturas da pele, levando consequentemente à quebra da sua integridade, com posterior formação de uma ferida evolutivamente exofítica, decorrente da proliferação celular descontrolada que o processo de oncogênese provoca ${ }^{4}$.

A prevalência dessas lesões, independente da localização anatômica, não é bem documentada, mas estima-se a partir de estudos internacionais que 5 a $10 \%$ dos pacientes com câncer desenvolvem feridas malignas ${ }^{4}$. Estudo ${ }^{5}$ realizado na Suíça, no período de seis meses, verificou que a prevalência de lesões malignas em pacientes com câncer metastático foi de $6,6 \%$.

Observa-se uma incipiência de dados epidemiológicos nacionais relacionados às feridas neoplásicas, por isso é difícil encontrar dados fidedignos no tocante à incidência de pessoas que apresentam diagnóstico de câncer e que progridem para o desenvolvimento destas lesões. Contudo, no Brasil, estudos quantitativos recentes abordaram dados sobre o acometimento de pessoas com neoplasias malignas, trazendo assim novas informações que evidenciam o perfil, características e tratamento das lesões neoplásicas ${ }^{6-7}$.

As feridas neoplásicas que acometem a pele constituem mais um agravo na vida do paciente oncológico, pois, progressivamente, desfiguram o corpo e tornam-se friáveis, dolorosas, exsudativas e liberam odor fétido ${ }^{8}$. Ao mesmo tempo, essas lesões podem levar ao desenvolvimento de complicações tais como infecções superficiais e/ou sistêmicas, fístulas e infestação de larvas; outrossim, essas feridas afligem também as dimensões psíquicas, sociais e espirituais do paciente, as quais podem interferir nas relações interpessoais com a equipe médica, com os próprios familiares e até mesmo o social ${ }^{2-4}$.

O tratamento das feridas neoplásicas malignas é complexo, pois exige avaliação de etiologia oncológica, características da ferida, estado físico e emocional do paciente e estadiamento da ferida. Diante disso, fazse necessário, que o enfermeiro, que habitualmente é responsável pela realização de curativos, tenha competência e habilidade para identificar, avaliar e tratar as feridas neoplásicas, proporcionando uma assistência integral ao paciente e sua família?.

Para proporcionar uma melhor qualidade de vida ao paciente com doença oncológica avançada com ferida neoplásica, o enfermeiro necessita conhecer produtos, substâncias, medicamentos e coberturas específicos para controle dos sinais e sintomas; assim como a realidade econômica do paciente e de seus familiares e da instituição em que está hospitalizado, para assim melhor intervir ${ }^{9}$.

Desta forma, cabe ao enfermeiro avaliar e tratar a lesão neoplásica levando em consideração as dimensões física, psíquica, social, espiritual e familiar do paciente, a fim de melhorar sua qualidade de vida durante seus últimos dias de vida, uma vez que o paciente que vive com doença oncológica avançada e apresenta ferida neoplásica possui um alto grau de vulnerabilidade física, psíquica e espiritual ${ }^{9}$.

Diante desse contexto, o enfermeiro frente ao paciente com ferida neoplásica, deve considerar durante a avaliação da lesão os seguintes aspectos: tamanho, profundidade, área de envolvimento, coloração, extensão, odor, exsudato, sangramento, dor, prurido, descamação, fístulas, abscessos, limitação física, metástases, adequação de roupas e curativos para o paciente ${ }^{10}$. Neste ínterim, o Instituto Nacional do Câncer (INCA) ${ }^{10}$ divulgou um manual de ações de enfermagem para pacientes com doença oncológica avançada que apresentam feridas neoplásicas, com o objetivo de uniformizar as intervenções avaliativas e terapêuticas para melhor cuidar destes pacientes.

A inquietação em realizar este estudo surgiu mediante visitas técnicas em um hospital filantrópico que atende pacientes com doença oncológica avançada onde foi possível verificar que os enfermeiros desconheciam o manual do 
INCA $^{10}$ e muitas vezes, utilizavam protocolos obsoletos na avaliação e tratamento de feridas. É imperioso destacar que se realizou uma busca nas bases de dados nacionais utilizando-se as palavras-chave: "feridas oncológicas", "feridas tumorais", "feridas neoplásicas", "feridas malignas", "conhecimento", "enfermeiros" e os operadores booleanos "OR" e "AND", e constatou-se uma escassez de artigos publicados que vislumbrassem esta temática.

Com base nesse caminhar, lançou-se a seguinte questão norteadora da pesquisa: qual o conhecimento e prática de enfermeiros no controle de ferida neoplásica? Nesse sentido, o objetivo geral deste estudo foi investigar o conhecimento e prática de enfermeiros no controle de feridas neoplásicas.

Esta pesquisa contribuirá na ampliação da produção científica relacionada à temática, disseminando o conhecimento entre os profissionais sobre a existência do manual do INCA e propor, a partir dos dados coletados, um protocolo específico para o cuidado a pacientes com feridas neoplásicas para a instituição locus da pesquisa.

\section{MÉTODO}

Trata-se de uma pesquisa descritiva de caráter quantitativo, realizada em um hospital filantrópico no município de Campina Grande/PB. A população definida para o estudo foi representada por enfermeiros assistenciais. Para selecionar a amostra, utilizou-se os seguintes critérios de inclusão: enfermeiros assistenciais, que realizassem curativos em pacientes com feridas neoplásicas com pelo menos seis meses de experiência na área, e que exercessem suas atividades laborais no dia da coleta. E como critérios de exclusão: enfermeiros que estivessem afastados no momento da coleta dos dados (licença saúde, férias, licença maternidade ou afastado para capacitação). Nesse sentido, participaram do estudo, 22 enfermeiros da referida instituição de saúde. A amostra foi não probabilística, pois considerou-se o sujeito que, consultado no local da unidade hospitalar dispusera em colaborar, respondendo ao questionário a ele apresentado pelo responsável da pesquisa.

Para coleta de dados foi elaborado um questionário estruturado, contendo duas seções: a primeira destinouse aos aspectos sociodemográficos e profissionais dos sujeitos da pesquisa, que teve como objetivo fazer uma breve caracterização dos participantes deste estudo; neste continha informações sobre o sexo, idade, estado civil, tempo de formação acadêmica e tempo de experiência na área de oncologia e pós-graduação. Na segunda parte do questionário foi elaborado um instrumento contendo questões acerca dos cuidados a pacientes com feridas neoplásicas, norteadas pelo manual do Ministério da Saúde e Instituto Nacional do Câncer sobre tratamento e controle de feridas tumorais - MS/INCA ${ }^{10}$ a saber:

A avaliação dos cuidados específicos com a ferida tumoral é um instrumento composto por cinco (5) subitens: a) controle do sangramento com 10 itens; b) controle do exsudato, composto por 3 itens; c) controle do odor, composto por 7 itens; d) controle da necrose, composto por 2 itens; f) controle da fístula cutânea, composto por 3 itens. Em todos eles, o respondente indicou, respectivamente, a sua resposta enfatizando o seu saber e fazer do cuidado com o paciente que apresentava feridas; para isso, ele indicou a sua resposta, referente ao saber, numa escala de três pontos ( 1 = não sei, $2=$ sei em parte e $3=$ sei). Em relação ao fazer, o mesmo sujeito assinalou as suas respostas numa escala binomial (por exemplo, $1=\operatorname{sim}$ e 2 = não).

A coleta foi realizada durante o período de maio a junho de 2016 e para a análise dos dados, a estatística descritiva (média, desvio padrão) utilizando o programa Statistical Package for the Social Sciences 22.0 (SPSS-22).

No que se refere às considerações éticas, a presente pesquisa foi norteada pelo Código de Ética dos Enfermeiros - Resolução $n^{0} 311 / 2007$ do Conselho Federal de Enfermagem, como também pelas Diretrizes e Normas Regulamentadoras para Pesquisa Envolvendo Seres Humanos, no cenário brasileiro, contemplados pela Resolução n 466/2012 do Conselho Nacional de Saúde (CNS)/Ministério da Saúde (MS) que dispõe sobre pesquisas com seres humanos.

Assim, o projeto de pesquisa foi ao Comitê de Ética em Pesquisa (CEP) do Hospital Universitário Alcides Carneiro, o qual teve sua aprovação com o parecer $n^{\circ} 1.321 .296$ e com CAAE nº 50354615.8.0000.5182.

\section{RESULTADOS E DISCUSSÃO}

Participaram do estudo, 22 enfermeiros da referida instituição de saúde, os quais, $91 \%$ eram do sexo feminino, $68 \%$ casados, com idade variando de 24 a 62 anos (Média = 36,6, dp = 9,31), 32\% tinham 5 anos de formação acadêmica, no que refere-se a experiência na área de oncologia, $54 \%$ afirmaram ter de 1 a 2 anos, $14 \%$ de 3 a 4 anos e de 5 a 10 anos $32 \%$ e $55 \%$ tinha pós-graduação lato sensu.

A formatação do banco de dados dessa pesquisa teve como objetivo avaliar a distribuição das respostas dos sujeitos nos referidos instrumentos sobre o cuidado com o paciente que apresentava ferida neoplásica. Desta forma, visando uma melhor compreensão para o leitor, os resultados serão apresentados por duas seções: a do saber e a do fazer das avaliações específicas destacadas no instrumento, isto é, apresentar-se-á inicialmente, o saber e o fazer dos cuidados específicos no que se refere ao controle do sangramento da ferida neoplásicas descritos na Tabela 1 logo a seguir e depois, as demais seções avaliativas:

Na Tabela 1, no que concerne à seção do saber, é possível observar que a maioria dos enfermeiros respondeu que sabiam, em parte, os cuidados específicos em relação ao sangramento na ferida neoplásica nos itens: considerar alginato de cálcio (45\%); considerar 
Tabela 1: Distribuição das respostas dos enfermeiros acerca do saber e do fazer sobre os cuidados específicos para o controle do sangramento realizados com a ferida neoplásica. Campina Grande - PB, Brasil, 2016.

Cuidados específicos que você realiza com a ferida neoplásica para o controle do sangramento

\begin{tabular}{|c|c|c|c|c|c|c|c|c|c|c|}
\hline & \multicolumn{6}{|c|}{ Quanto você sabe } & \multicolumn{4}{|c|}{ Quanto você faz } \\
\hline & \multicolumn{2}{|c|}{ Não sei } & \multicolumn{2}{|c|}{ Sei em parte } & \multicolumn{2}{|c|}{ Sei } & \multicolumn{2}{|c|}{ Sim } & \multicolumn{2}{|c|}{ Não } \\
\hline & $\mathrm{n}$ & $\%$ & $\mathrm{n}$ & $\%$ & $\mathrm{n}$ & $\%$ & $\mathrm{n}$ & $\%$ & $\mathrm{n}$ & $\%$ \\
\hline $\begin{array}{l}\text { 1.Aplicar pressão diretamente sobre os vasos } \\
\text { sangrantes com amparo de gazes, compressas ou } \\
\text { toalhas }\end{array}$ & 2 & 9 & 4 & 18 & 16 & 73 & 16 & 73 & 6 & 27 \\
\hline 2.Considerar aplicação de solução salina gelada & 2 & 9 & 7 & 32 & 13 & 59 & 13 & 59 & 9 & 41 \\
\hline $\begin{array}{l}\text { 3.Considerar curativo hemostático à base de } \\
\text { gelatina suína (Gelfoam) }\end{array}$ & 13 & 59 & 5 & 23 & 4 & 18 & 1 & 5 & 21 & 95 \\
\hline 4.Considerar alginato de cálcio & 9 & 41 & 10 & 45 & 3 & 14 & 2 & 9 & 20 & 91 \\
\hline $\begin{array}{l}\text { 5.Considerar a aplicação de adrenalina (solução } \\
\text { injetável) topicamente sobre os pontos sangrantes } \\
\text { conforme prescrição médica }\end{array}$ & 6 & 27 & 9 & 41 & 7 & 32 & 5 & 23 & 17 & 77 \\
\hline $\begin{array}{l}\text { 6.Manter o meio úmido evitando aderência de gaze } \\
\text { no leito da ferida }\end{array}$ & 3 & 14 & 4 & 18 & 15 & 68 & 12 & 55 & 10 & 45 \\
\hline $\begin{array}{l}\text { 7.Verificar, junto à equipe médica, a possibilidade de } \\
\text { tratamento com coagulante sistêmico como ácido } \\
\text { aminocaproico }\end{array}$ & 11 & 50 & 8 & 36 & 3 & 14 & 2 & 9 & 20 & 91 \\
\hline $\begin{array}{l}\text { 8.Verificar, junto à equipe médica, a possibilidade de } \\
\text { tratamento com intervenção cirúrgica }\end{array}$ & 6 & 27 & 10 & 46 & 6 & 27 & 5 & 23 & 17 & 77 \\
\hline $\begin{array}{l}\text { 9.Verificar, junto à equipe médica, a possibilidade de } \\
\text { tratamento com radioterapia anti-hemorrágica }\end{array}$ & 8 & 36 & 11 & 50 & 3 & 14 & 4 & 18 & 18 & 82 \\
\hline $\begin{array}{l}\text { 10.Verificar, junto à equipe médica, a possibilidade } \\
\text { de tratamento com sedação paliativa para os casos } \\
\text { de sangramento intenso acompanhado de agitação, } \\
\text { desespero e angústia do paciente }\end{array}$ & 7 & 32 & 9 & 41 & 6 & 27 & 4 & 18 & 18 & 82 \\
\hline TOTAL & 22 & 100 & 22 & 100 & 22 & 100 & 22 & 100 & 22 & 100 \\
\hline
\end{tabular}

Fonte: dados da pesquisa, 2016.

adrenalina (solução injetável) topicamente sobre os pontos sangrantes conforme prescrição médica (41\%); verificar, junto à equipe médica, a possibilidade de tratamento com intervenção cirúrgica (46\%); verificar, junto à equipe médica, a possibilidade de tratamento com radioterapia anti-hemorrágica (50\%) e verificar, junto à equipe médica, a possibilidade de tratamento com sedação paliativa (41\%) para os casos de sangramento intenso acompanhado de agitação, desespero e angústia do paciente e a maioria declarou não saber considerar curativo hemostático à base de gelatina suína (Gelfoam) (59\%) para controle do sangramento.

No que refere-se à seção do fazer, a maioria dos sujeitos (91\%) respondeu que não fazia os cuidados específicos em relação ao sangramento na ferida neoplásica.

Conforme os resultados apontados na Tabela 1, pode-se perceber, mais uma vez, um conhecimento insuficiente dos sujeitos da pesquisa acerca do controle dos sintomas das feridas neoplásicas, envolvendo o manejo do sangramento. Esse é um dado que merece bastante atenção, uma vez que a hemorragia pode causar sensação de ansiedade, inquietação e angústia ao paciente, principalmente, àquele que tem fobia; mas, sobretudo, pode ocasionar alterações hemodinâmicas severas, levando-o a óbito ${ }^{2-8-9}$.
As hemorragias tumorais, geralmente, são as formas mais intensas de sangramento e de difícil manejo, por isso,é fundamental a localização precisa do sítio do sangramento, associada à definição do estadiamento do tumor, para que se possa tomar a conduta mais adequada ${ }^{2-4-11}$.

Nestas circunstâncias, é recomendado para a prevenção do sangramento, prioritariamente, o uso de coberturas não aderentes, tais como as de silicone, uma vez que evitam aderência sobre o leito da lesão e possível sangramento durante as trocas de curativos. Os curativos não aderentes são aplicados diretamente sobre o leito da lesão e não causam traumas significativos no momento da remoção, contudo exigem cobertura secundária. Outros fatores importantes que devem ser ressaltados para a prevenção do sangramento dizem respeito à irrigação com solução salina do curativo anterior (quando for utilizado coberturas aderentes) e limpeza da ferida com técnica de irrigação $0^{2-4-8-9,11}$.

Para o controle do sangramento, uma variedade de hemostáticos pode ser aplicada topicamente ou sistemicamente para controlar a hemorragia. Esses agentes hemostáticos variam de acordo com a aplicação e mecanismos. Exemplos incluem: hemostáticos naturais (alginatos de cálcio, colágeno e celulose oxidada), 
coagulantes (gelatina em pó absorvível ou trombina tópica), agentes esclerosantes (nitrato de prata, ácido tricloroacético), vasoconstritores (epinefrina), inibidores (ácido tranexâmico, fibrinolíticos) e adstringentes (solução de alumínio e sucralfato) $)^{2-4-8-9-11}$.

Outras modalidades farmacológicas que podem ser utilizadas pela equipe são o ácido aminocaproico e o ácido tranexâmico, os quais agem sistematicamente; já as suturas, pinçamentos, cauterizações constituem intervenções cirúrgicas hemostáticas locais, geralmente

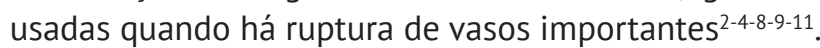

A radioterapia paliativa objetiva o tratamento local do tumor primário ou de metástases, sem influenciar na sobrevida do paciente e é usada principalmente para o controle da dor e do sangramento. As indicações da radioterapia anti-hemorrágica incluem controle de sangramento bucal, epistaxe, metrorragia, hematúria, sangramento vaginal e retal; além disso, é usada também para controlar o crescimento rápido de tumores exofíticos ${ }^{2-4-11}$.

Estudo $^{12}$ realizado com 32 mulheres com feridas vegetantes mamárias, constatou algumas medidas realizadas para redução do sangramento, dentre elas: remoção do curativo de forma delicada, utilização de coberturas antiaderentes (silicone), alginato como curativo hemostático e adrenalina como vasoconstritor local e sistêmico.

Um sangramento maciço é muito doloroso para o paciente e sua família, não só por causa da hemorragia, mas, sobretudo porque a perda profusa de sangue pode causar a morte iminente do paciente. Neste sentido, um plano estratégico deve ser desenvolvido em conjunto com o paciente e família quando um episódio de sangramento maciço acontecer: explicação acerca da possibilidade de hemorragia profusa para cuidadores e familiares; orientações acerca das atitudes que devem ser realizadas pela família no momento da hemorragia; deixar próximo à cabeceira do paciente, toalhas de cores escuras; preparação de um kit de emergência, incluindo droga sedativa; acesso ao serviço de transporte de emergência e linhas de comunicação abertas com centros especializados apropriados, nas quais a emergência pode ser rapidamente chamada ${ }^{5-8}$.

Na Tabela 2, no que concerne à seção do saber, é possível observar, que os a maioria dos enfermeiros respondeu que sabia, em parte, os cuidados específicos para o controle do exsudato na ferida neoplásica nos itens: coletar material para cultura (aspirado ou swab) (41\%); empregar carvão ativado/ alginato de cálcio e compressas/gaze como cobertura secundária (54\%); por outro lado, no item empregar óxido de zinco na pele macerada e nas bordas da ferida, os enfermeiros indicaram que não sabiam (50\%) realizar esse cuidado.

No que refere-se à seção do fazer, todos os sujeitos (100\%) responderam que não faziam os cuidados específicos para o controle do exsudato na ferida neoplásica.

De acordo com os resultados da Tabela 2 é possível verificar novamente que os enfermeiros da pesquisa apresentam fragilidades constantes no que refere-se ao conhecimento e prática do cuidado com pacientes com feridas neoplásicas. Esse é um dado preocupante, pois as feridas neoplásicas apresentam quantidade excessiva de exsudato, o que desencadeia várias complicações, dentre elas o odor ${ }^{5-8-9}$.

As feridas neoplásicas, geralmente, apresentam grandes quantidades de exsudato e quando não controlado de forma eficaz, pode servir como meio de cultura para proliferação de micro-organismos, o que concorre para o desenvolvimento de odores; maceração da pele periferida, que por sua vez, contribui para a extensão da lesão; além disso, quantidades excessivas de exsudato podem ultrapassar as coberturas primária e secundária, sujando as roupas do paciente, causando problemas psicossociais significativos para o mesmo. 0 controle desse sintoma reduz o odor, evita sujar as roupas do paciente e da cama, com vistas à proporcionar conforto e confiança ao mesmo ${ }^{5-8-9}$.

A característica do exsudato presente nas feridas é um importante indicador que auxilia no diagnóstico clínico de

Tabela 2: Distribuição das respostas dos enfermeiros acerca do saber e do fazer sobre os cuidados específicos para o controle do exsudato realizados com a ferida neoplásica. Campina Grande - PB, Brasil, 2016.

\begin{tabular}{|c|c|c|c|c|c|c|c|c|c|c|}
\hline \multicolumn{11}{|c|}{ Cuidados específicos que você realiza com a ferida neoplásica para o controle do exsudato } \\
\hline & \multicolumn{6}{|c|}{ Quanto você sabe } & \multicolumn{4}{|c|}{ Quanto você faz } \\
\hline & \multicolumn{2}{|c|}{ Não sei } & \multicolumn{2}{|c|}{ Sei em parte } & \multicolumn{2}{|c|}{ Sei } & \multicolumn{2}{|c|}{ Sim } & \multicolumn{2}{|c|}{ Não } \\
\hline & $\mathrm{n}$ & $\%$ & $\mathrm{n}$ & $\%$ & $\mathrm{n}$ & $\%$ & $\mathrm{n}$ & $\%$ & $\mathrm{n}$ & $\%$ \\
\hline 1.Coletar material para cultura (aspirado e swab) & 7 & 32 & 9 & 41 & 6 & 27 & 1 & 5 & 21 & 95 \\
\hline $\begin{array}{l}\text { 2.Empregar carvão ativado/alginato de cálcio } \\
\text { como cobertura primária e compressas/gazes como } \\
\text { cobertura secundária }\end{array}$ & 6 & 27 & 12 & 54 & 4 & 18 & 2 & 9 & 20 & 91 \\
\hline $\begin{array}{l}\text { 3.Empregar óxido de zinco nas bordas da pele } \\
\text { periferida }\end{array}$ & 11 & 50 & 7 & 32 & 4 & 18 & 2 & 9 & 20 & 91 \\
\hline TOTAL & 22 & 100 & 22 & 100 & 22 & 100 & 22 & 100 & 22 & 100 \\
\hline
\end{tabular}

Fonte: dados da pesquisa, 2016. 
infecção e escolha de terapias tópicas. Para isso, alguns métodos de avaliação ajudam a quantificar e classificar o tipo de exsudato da lesão, a saber: Escala de Bates - Jensen e Sistema TELER ${ }^{13}$. Método padrão-ouro para analisar a carga microbiana é a cultura de secreção de tecido viável da ferida por meio de uma biópsia e o método de Levine ${ }^{14}$.

Nessas circunstâncias, o tipo de cobertura a ser escolhido dependerá das especificidades da lesão, tais como localização, dimensões, estadiamento e características dos tecidos. Para o controle do exsudato convém utilizar coberturas absorventes, espuma de prata, hidropolímeros, carvão ativado e alginato ${ }^{5-8-9}$. Como cobertura secundária, comumente, utiliza-se gaze dupla, aberta estéril, compressa cirúrgica associada à atadura e algum tipo de cobertura impermeável, como filme de poliuretano a fim de ocluir e fixar a cobertura primária2-4-8

A pele periferida é aquela que encontra-se circunscrita em torno da lesão, com uma extensão que varia de acordo com a etiologia,localização,tipo,características e magnitude da ferida. Na maioria das vezes, essa pele está exposta à ação de fluidos e exsudatos provenientes da própria lesão e pode apresentar-se fria, seca, fina, descamativa, hiperpigmentada, macerada e com dermatites. As bordas da ferida, do mesmo modo, sofrem essas repercussões e pode apresentar-se com aspecto regular ou irregular, aderido ou não, espesso e friável ou com cicatriz fibrótica. Por esses motivos, a pele perilesional e borda merecem os mesmos cuidados que o leito da lesão, de acordo com as suas especificidades ${ }^{4}$. Neste sentido, autores ${ }^{2-4-5}$ ressaltam a importância do uso de pomada a base de óxido de zinco como profilaxia em relação à toxicidade do exsudato na região periferida, bordas da lesão e pele íntegra.

Pesquisa ${ }^{12}$ realizada com 32 mulheres com feridas neoplásicas mamárias, verificou que as amostras microbiológicas foram obtidas utilizando uma cureta em cada avaliação, a fim de identificar e quantificar bactérias aeróbias e anaeróbias e caracterizar a presença de biofilme sob fluorescência após coloração. $O$ exsudato foi controlado usando como coberturas primárias alginato, hidrofibra e/ou hidrocelular e como coberturas secundárias, compressas cirúrgicas.

Vale ressaltar que curativos altamente absorventes proporcionam maior conforto e segurança para os pacientes com feridas neoplásicas, uma vez que o extravasamento de fluidos apresenta impacto no bem-estar emocional, gerando constrangimento aos mesmos ${ }^{2-4-8}$.

Assim, pode-se constatar, novamente, que os enfermeiros desta pesquisa apresentam uma lacuna no conhecimento acerca das técnicas de avaliação, produtos e coberturas utilizadas no cuidado a pacientes com feridas neoplásicas, o que contribui para uma assistência de saúde frágil.

Na Tabela 3, no que concerne à seção do saber,é possível observar que a maioria dos enfermeiros afirmou que não sabia os cuidados específicos em relação ao odor na ferida neoplásica nos itens: empregar sulfadiazina de prata em

Tabela 3: Distribuição das respostas dos enfermeiros acerca do saber e do fazer sobre os cuidados específicos para o controle do odor realizados com a ferida neoplásica. Campina Grande - PB, Brasil, 2016.

\begin{tabular}{|c|c|c|c|c|c|c|c|c|c|c|}
\hline \multicolumn{11}{|c|}{ Cuidados específicos que você realiza com a ferida neoplásica para o controle do odor } \\
\hline & \multicolumn{6}{|c|}{ Quanto você sabe } & \multicolumn{4}{|c|}{ Quanto você faz } \\
\hline & \multicolumn{2}{|c|}{ Não sei } & \multicolumn{2}{|c|}{ Sei em parte } & \multicolumn{2}{|c|}{ Sei } & \multicolumn{2}{|c|}{ Sim } & \multicolumn{2}{|c|}{ Não } \\
\hline & $\mathrm{n}$ & $\%$ & $\mathrm{n}$ & $\%$ & $\mathrm{n}$ & $\%$ & $\mathrm{n}$ & $\%$ & $\mathrm{n}$ & $\%$ \\
\hline $\begin{array}{l}\text { 1.Proceder à limpeza com solução salina e realizar } \\
\text { antissepsia com clorohexidina }\end{array}$ & 1 & 5 & 6 & 27 & 15 & 68 & 18 & 82 & 4 & 18 \\
\hline $\begin{array}{l}\text { 2.Retirar antisséptico com jato de solução salina e } \\
\text { depois manter gazes embebidas em hidróxido de } \\
\text { alumínio no leito da ferida }\end{array}$ & 6 & 27 & 9 & 41 & 7 & 32 & 4 & 18 & 18 & 82 \\
\hline $\begin{array}{l}\text { 3.Empregar sulfadiazina de prata no leito da lesão e } \\
\text { depois ocluir com gaze embebida em vaselina líquida }\end{array}$ & 11 & 50 & 6 & 27 & 5 & 23 & 3 & 14 & 19 & 86 \\
\hline $\begin{array}{l}\text { 4.Empregar carvão ativado no leito da lesão e cobrir } \\
\text { com gaze umedecida com solução salina }\end{array}$ & 10 & 46 & 6 & 27 & 5 & 23 & 2 & 9 & 20 & 91 \\
\hline $\begin{array}{l}\text { 5.Aplicar gel de metronidazol } 0,8 \% \text { conforme } \\
\text { prescrição médica, para odor grau I, no leito da ferida } \\
\text { e depois cobrir com em gaze embebida em vaselina }\end{array}$ & 7 & 32 & 3 & 14 & 12 & 55 & 11 & 50 & 11 & 50 \\
\hline $\begin{array}{l}\text { 6.Se houver necessidade, fazer escarotomia em } \\
\text { tecido necrótico e proceder à aplicação de gel de } \\
\text { metronidazol, conforme prescrição médica, para odor } \\
\text { grau II }\end{array}$ & 10 & 46 & 11 & 50 & 1 & 5 & 1 & 5 & 21 & 95 \\
\hline $\begin{array}{l}\text { 7.Considerar junto à equipe médica, a possibilidade } \\
\text { de associação de metronidazol sistêmico } \\
\text { (endovenoso ou oral) ao uso tópico, para odor grau III }\end{array}$ & 12 & 55 & 6 & 27 & 4 & 18 & 2 & 9 & 20 & 91 \\
\hline TOTAL & 22 & 100 & 22 & 100 & 22 & 100 & 22 & 100 & 22 & 100 \\
\hline
\end{tabular}

Fonte: dados da pesquisa, 2016. 
gaze e ocluir com gaze embebida em vaselina líquida (50\%); empregar carvão ativado em gaze umedecida com soro fisiológico a $0,9 \%$, se houver necessidade (46\%); fazer escarotomia em tecido necrótico e proceder à aplicação de gel de metronidazol conforme orientação médica (46\%) e considerar junto à equipe médica, a possibilidade de associação de metronidazol sistêmico (endovenoso ou oral) ao uso tópico (55\%). No item 'retirar antisséptico com jato de soro fisiológico a $0,9 \%$ e manter gazes embebidas em hidróxido de alumínio no leito da ferida', a maioria dos enfermeiros (91\%) respondeu que sabia.

No que refere-se à seção do fazer, a maioria dos sujeitos respondeu que não fazia os cuidados específicos em relação ao odor na ferida neoplásica nos itens: retirar antisséptico com jato de soro fisiológico a $0,9 \%$ e manter gazes embebidas em hidróxido de alumínio no leito da ferida (82\%); empregar sulfadiazina de prata em gaze e ocluir com gaze embebida em vaselina líquida (86\%); empregar carvão ativado em gaze umedecida com soro fisiológico a $0,9 \%$, se houver necessidade (91\%); fazer escarotomia em tecido necrótico e proceder à aplicação de gel de metronidazol conforme prescrição médica e considerar junto à equipe médica, a possibilidade de associação de metronidazol sistêmico (endovenoso ou oral) ao uso tópico (91\%). No que concerne ao fazer, a intervenção que revelou prática da maioria dos enfermeiros (82\%) foi proceder à limpeza com solução salina e realizar antissepsia com clorohexidina.

Nesta mesma seção vale destacar que, no item aplicar gel de metronidazol 0,8\% conforme prescrição médica em gaze embebida em vaselina e aplicar no leito da ferida, o resultado foi igualitário para o estudo, levando a uma ambiguidade a respeito da frequência.

É possível perceber a repetição dos resultados nas seções saber e fazer do estudo, visto que a maioria dos enfermeiros desconhece as medidas utilizadas para 0 controle de sintomas das feridas neoplásicas, em particular, - odor, ressaltado na Tabela destacada anteriormente, revelando uma lacuna no âmbito da educação em saúde.

O odor fétido é considerado pelos pacientes como o sintoma mais angustiante, pois leva ao constrangimento, sensação de enojamento, depressão, isolamento social e efeito prejudicial sobre a sexualidade, causando problemas no relacionamento. O odor é detectado por receptores olfativos situados na região nasal e é processado tanto a nível consciente e subconsciente, desencadeando reflexos de vômitos. A nível subconsciente, o odor fétido afeta a experiência de sabor e aroma. Isso, obviamente, tem um impacto profundamente negativo sobre o paciente, diminuindo o seu apetite e prazer pela comida, o que contribui para emagrecimento acentuado ${ }^{4-8-9}$. O odor é um sintoma que é difícil de avaliar. Autores ${ }^{15}$ descreveram um indicador clínico para quantificar odor, cujo objetivo é determinar o impacto desse sintoma na vida da pessoa com esse tipo de lesão. Por esse motivo, o Instituto Nacional do Câncer $^{10}$, baseado no sistema TELER ${ }^{13}$, construiu uma escala de avaliação de odor para feridas neoplásicas, que o classifica em três níveis, a saber: grau I, II e III.

Para o tratamento do odor, o primeiro passo é realizar a limpeza da lesão com antisséptico, tais como a clorexidina, pois pertence ao grupo das biguanidas e age sobre os micro-organismos gram-positivos e gram-negativos ${ }^{4-15-16}$. Como coberturas primárias, pode-se utilizar sulfadiazina de prata a $1 \%$ e metronidazol em várias apresentações.

Autores $^{4-14}$ recomendam algumas ações que devem ser valorizadas pelos profissionais de saúde no controle de infecções de feridas, como: considerar a utilização de antissépticos tópicos em conjunto com os desbridamentos de manutenção a fim de controlar e erradicar o biofilme que se suspeita estar presente nas feridas de difícil cicatrização; considerar a utilização de antissépticos tópicos nas feridas e que se encontram altamente colonizadas/infectadas e considerar a utilização de sulfadiazina de prata em feridas altamente contaminadas ou infectadas até que seja realizado o desbridamento definitivo. Além dessas modalidades farmacológicas, os pacientes beneficiam-se também com o uso de compressas embebidas de hidróxido de alumínio ${ }^{10}$.

Caso o paciente apresente uma lesão com tecido necrótico de aspecto endurecido, inelástico e crosta, que compromete o enchimento capilar, a irrigação sanguínea, prejudicando a oxigenação tissular, o profissional pode proceder com a técnica de Square (escarotomia) sobre a ferida. Além de melhorar a perfusão sanguínea, a escarotomia em feridas necrosadas facilita a penetração dos produtos na ferida, o que contribui para absorção ${ }^{16}$.

Estudo $^{12}$ realizado com 32 mulheres com feridas vegetantes mamárias, constatou algumas medidas realizadas para redução do odor, dentre elas: limpeza da lesão com soluções à base de polihexanidametil biguanida (PHMB); coberturas primárias como carvão ativado, impregnados de prata, hidrocelululares contendo nanocristais de prata e antimicrobianos tópicos como o metronidazol.

O odor é um problema grave que afeta tanto a imagem corporal como a qualidade de vida dos pacientes com feridas neoplásicas, assim como aqueles que estão em seu entorno. Por esse motivo, os enfermeiros precisam estar adequadamente instruídos e preparados para o controle desse sintoma e para intervir frente às demandas emocionais que o paciente venha apresentar.

$\mathrm{Na}$ Tabela 4, no que concerne à seção do saber, é possível observar que a maioria dos enfermeiros respondeu que sabia, em parte, os cuidados específicos em relação ao controle da necrose na ferida neoplásica nos itens: avaliar as necessidades de desbridamento, de acordo com a capacidade do paciente (50\%) e eleger a forma de desbridamento (mecânico, químico, autolítico) (55\%). Já na seção do fazer, a maioria dos sujeitos respondeu (73\%) que não fazia os cuidados específicos em relação ao controle da necrose na ferida neoplásica. 
Tabela 4: Distribuição das respostas dos enfermeiros acerca do saber e do fazer sobre os cuidados específicos realizados para o controle da necrose da ferida neoplásica. Campina Grande - PB, Brasil, 2016.

\begin{tabular}{|c|c|c|c|c|c|c|c|c|c|c|}
\hline \multicolumn{11}{|c|}{ Cuidados específicos que você realiza com a ferida neoplásica para o controle da necrose } \\
\hline & \multicolumn{6}{|c|}{ Quanto você sabe } & \multicolumn{4}{|c|}{ Quanto você faz } \\
\hline & \multicolumn{2}{|c|}{ Não sei } & \multicolumn{2}{|c|}{ Sei em parte } & \multicolumn{2}{|c|}{ Sei } & \multicolumn{2}{|c|}{ Sim } & \multicolumn{2}{|c|}{ Não } \\
\hline & $\mathrm{n}$ & $\%$ & $\mathrm{n}$ & $\%$ & $\mathrm{n}$ & $\%$ & $\mathrm{n}$ & $\%$ & $\mathrm{n}$ & $\%$ \\
\hline $\begin{array}{l}\text { 1.Avaliar as necessidades de desbridamento, de } \\
\text { acordo com a capacidade do paciente }\end{array}$ & 3 & 14 & 11 & 50 & 8 & 36 & 6 & 27 & 16 & 73 \\
\hline $\begin{array}{l}\text { 2.Eleger a forma de debridamento (mecânico, } \\
\text { químico, autolítico) }\end{array}$ & 3 & 14 & 12 & 55 & 7 & 54 & 7 & 32 & 15 & 68 \\
\hline TOTAL & 22 & 100 & 22 & 100 & 22 & 100 & 22 & 100 & 22 & 100 \\
\hline
\end{tabular}

Fonte: dados da pesquisa, 2016.

Segundo os resultados mencionados na Tabela 4, podese perceber, mais uma vez, um conhecimento insuficiente dos sujeitos da pesquisa acerca do controle dos sintomas das feridas neoplásicas; agora, envolvendo o manejo da necrose.

Em tratando-se do cuidado com a necrose, a melhor conduta é o desbridamento autolítico e enzimático, mas deve-se avaliar os riscos e os benefícios deste procedimento diante do paciente com ferida neoplásica maligna, uma vez que a friabilidade das lesões pode ocasionar o risco potencial para sangramentos maciços s-5-11-16. $^{\text {. }}$

Neste sentido, alguns fatores devem ser levados em consideração antes da realização do desbridamento, tais como: a área a ser desbridada, a presença de infecções locais, a vascularização e neovascularização do local, os riscos de sangramento durante ou após o procedimento, controle de odor resistente às terapias prévias e a condição geral do paciente -5-51-16. $^{-16}$

Nestas feridas, a retirada da debris pode ser realizada por meio de manutenção de meio úmido, pela limpeza suave das áreas necróticas ou utilizando jatos de solução salina a pressão de 8 a 15 psi (pound force per square $(i n c h)^{17}$. Quando o tumor apresenta tecido necrosado extenso, o desbridamento cirúrgico pode ser indicado a fim de prevenir a infecção, controle do exsudato e odor, no entanto, há de se ponderar os casos em que o paciente se encontra em cuidados paliativos, tendo em vista que tal procedimento gera maior sofrimento físico e emocional ao mesmo e à família ${ }^{11}$.

Pesquisa ${ }^{12}$ realizada com 20 enfermeiras na mesma instituição que foi realizada o estudo, verificou que as participantes da pesquisa mencionaram que o serviço onde as mesmas realizavam os cuidados com feridas neoplásicas não disponibiliza materiais e/ou curativos específicos para o controle da necrose, ficando a família - apenas, aquela que apresentar condições favoráveis e financeiras - responsável pela compra de tais produtos. $\mathrm{Na}$ direção desse resultado, aponta-se para uma política brasileira de saúde frágil e limitada psicossocialmente, principalmente para pacientes em terminalidade.

Estudo ${ }^{18}$ de intervenção realizado com 12 mulheres com feridas malignas constatou o benefício do desbridamento autolítico em relação ao cirúrgico. Os curativos eram realizados com hidrogel como cobertura primária e espuma como cobertura secundária. Os resultados apontaram os seguintes achados: 09 mulheres (75\%), após o período de intervenção, apresentaram diminuição do tamanho da lesão e aparecimento de tecido de granulação e epitelização; 01 paciente teve sua lesão totalmente cicatrizada e em 03 pacientes (25\%), as feridas aumentaram de tamanho e apresentaram tecido de granulação, bem como quantidade expressiva de fibrose, necrose amarela e preta, além de infecção. Isso pode ter sido porque essas 03 mulheres não estavam recebendo tratamento antineoplásico ${ }^{18}$.

A cicatrização de feridas tumorais malignas do estudo supracitado foi observada durante o período de intervenção, um achado não relatado, até onde se sabe, na literatura. Em alguns estudos autores ${ }^{4-5,11,16}$ afirmam que a cura de feridas fungosas malignas é uma meta irrealista, e que o cuidado da ferida é unicamente paliativo. Portanto, é importante afirmar que, através desta intervenção, os pesquisadores conseguiram otimizar a cicatrização, com aumento da vascularização, granulação e epitelização e redução do tamanho da ferida. De forma a atualizar tais dados, a EPUAP e NPUAP recomendam aos profissionais de saúde considerar a utilização de hidrogel para tratamento de feridas que apresentam leitos secos ${ }^{5}$.

Estudo $^{19}$ realizado com 04 pacientes com doença oncológica verificou a eficácia da pasta de mel de Leptospermum ativo (ALH) nas lesões malignas. Observou-se cicatrização total das lesões em 02 pacientes e nos outros 02 houve redução da intensidade da dor e do tamanho da lesão, erradicação do odor, diminuição de tecido de liquefação e aumento de tecido de granulação. Apesar de ser um relato de experiência ${ }^{15}$, a EPUAP e NPUAP recomendam aos profissionais de saúde considerar a utilização de mel em feridas altamente contaminadas ou infectadas até que seja realizado o desbridamento definitivo.

Na Tabela 5 , no que concerne à seção do saber,é possível observar que a maioria dos enfermeiros respondeu que sabia, em parte, os cuidados específicos em relação ao controle da fístula cutânea na ferida neoplásica nos itens: aplicar óxido de zinco na pele ao redor da fístula (55\%); adaptar, quando possível, uso de bolsas coletoras nas fístulas de alta drenagem, com placas de hidrocoloide ao 
Tabela 5: Distribuição das respostas dos enfermeiros acerca do saber e do fazer sobre os cuidados específicos realizados para o controle da fístula cutânea em ferida neoplásica. Campina Grande - PB, Brasil, 2016.

\begin{tabular}{|c|c|c|c|c|c|c|c|c|c|c|}
\hline \multicolumn{11}{|c|}{ Cuidados específicos que você realiza com a ferida neoplásica para o controle da fístula cutânea } \\
\hline & \multicolumn{6}{|c|}{ Quanto você sabe } & \multicolumn{4}{|c|}{ Quanto você faz } \\
\hline & \multicolumn{2}{|c|}{ Não sei } & \multicolumn{2}{|c|}{ Sei em parte } & \multicolumn{2}{|c|}{ Sei } & \multicolumn{2}{|c|}{ Sim } & \multicolumn{2}{|c|}{ Não } \\
\hline & $\mathrm{n}$ & $\%$ & $\mathrm{n}$ & $\%$ & $\mathrm{n}$ & $\%$ & $\mathrm{n}$ & $\%$ & $\mathrm{n}$ & $\%$ \\
\hline 1.Aplicar óxido de zinco na pele ao redor da fístula & 4 & 18 & 12 & 55 & 6 & 27 & 3 & 14 & 19 & 86 \\
\hline $\begin{array}{l}\text { 2.Adaptar, quando possível, uso de bolsas coletoras } \\
\text { nas fístulas de alta drenagem e placas de } \\
\text { hidrocoloide ao redor da pele }\end{array}$ & 4 & 18 & 10 & 46 & 8 & 36 & 8 & 36 & 14 & 64 \\
\hline $\begin{array}{l}\text { 3.Realizar curativo absortivo com carvão ativado e/ } \\
\text { ou alginato de cálcio como cobertura primária e } \\
\text { compressa/gaze como cobertura secundária }\end{array}$ & 4 & 18 & 12 & 54 & 6 & 28 & 5 & 23 & 17 & 77 \\
\hline TOTAL & 22 & 100 & 22 & 100 & 22 & 100 & 22 & 100 & 22 & 100 \\
\hline
\end{tabular}

Fonte: dados da pesquisa, 2016.

redor da pele (46\%) e realizar curativo absortivo com carvão ativado e/ou alginato de cálcio como cobertura primária e compressa/gaze como cobertura secundária (54\%). E na seção fazer, a maioria dos sujeitos (86\%) respondeu que não fazia os cuidados específicos em relação ao controle da fistula cutânea na ferida neoplásica.

Novamente, os dados coadunam para uma prática assistencial de enfermagem frágil, lacunar e incipiente, o que remete a necessidade de criação e implementação de educação permanente no serviço.

A fístula cutânea pode surgir em decorrência de complicações de tratamentos como cirurgias, braquiterapia, radioterapia, ou ainda, avanço e/ou recidiva da doença. Geralmente, a formação de fístulas em feridas tumorais malignas está diretamente relacionada à progressão do câncer. Os principais tipos de cânceres que têm maior probabilidade de formar fístulas são os de cabeça e pescoço (fístulas oro-cutâneas e esôfago-cutâneas) e o ginecológico (fístula externa, denominada cutânea e fístula interna). A presença de fístulas agrava a ferida, tornando-a mais exsudativa e fétida, bem como ocasiona erosão da pele periferida, tornando o paciente mais vulnerável ao isolamento social ${ }^{20-21}$.

Os cuidados de enfermagem relacionados à presença de fístulas na ferida tumoral maligna são, prioritariamente, avaliação do trajeto e estado da fístula, tratamento, educação do paciente e seu cuidador. Um dos cuidados essenciais diz respeito à avaliação do efluente no que refere-se a coloração, presença de odor e volume drenado nas 24 horas. De acordo com o volume do exsudato, a fístula pode ser classificada em baixo débito (menos de $500 \mathrm{~mL}$ ) e alto débito (acima de $500 \mathrm{~mL}$ ). Os cuidados no manejo das fístulas devem considerar que, se há débito de até 250 $\mathrm{mL} / 24$ horas, o mesmo poderá ser contido com gazes e/ou curativos altamente absorventes; em drenagens de maior volume, indica-se a colocação de coletores para estomias, inclusive os pediátricos, que são menores e se adaptam melhor ao corpo do paciente. Devido às características irregulares na forma dessas feridas, esses produtos podem ser úteis para moldar as bordas da lesão, de forma a permitir que a bolsa coletora seja acoplada para receber o efluente. É imprescindível a proteção da pele ao redor da fístula, e para isso, pode ser realizada a aplicação de placas de hidrocoloide, selantes, creme e/ou pasta barreira ${ }^{20}$.

Quando as fístulas apresentam odor, deve-se considerar o uso de carvão ativado e/ou cápsulas gelificantes para colostomias (que se desintegram no meio líquido e controlam o odor dos efluentes). Outrossim, o enfermeiro e a equipe médica podem discutir sobre a possibilidade do uso de metronidazol sistêmico diante de odores mais ofensivos ou outros fármacos, como hioscina e octreotide, que também são recomendados na assistência paliativa. A pessoa com feridas em estágios avançados com risco de desenvolver fístulas, geralmente, encontra-se desnutrida, apresenta hipoalbuminemia $(<20 \mathrm{mg} / \mathrm{dL}$ ) ou balanço negativo de proteínas, anemia significativa $(\mathrm{Hb}<8)$ e utiliza altas doses de drogas imunossupressoras. Por esse motivo, considerando, integralmente, o entorno da pessoa afetada pelo câncer avançado e pela presença da ferida neoplásica, a avaliação do estado nutricional e seu balanço hidroeletrolítico por meio de exames laboratoriais são mandatórios ${ }^{20}$.

Urge mencionar que, ao fim da coleta, os enfermeiros reconheceram verbalmente que não imaginavam o conhecimento necessário na alternativa de cada item e que, no início, atribuíram um domínio de conhecimento equivocado. A autoavaliação foi um importante método de auto-observação para que esses profissionais pudessem identificar suas carências, lacunas e fragilidades, o que estimula um diálogo interno construtivo. Todavia, esse exercício somente será válido para os que se autorregularem e se modificarem. Deste modo, identificar as necessidades de aprendizagem é tão importante quando a vontade de modificar suas práticas.

\section{CONCLUSÃO}

A análise evidenciou que os enfermeiros apresentam limitações no conhecimento para avaliar as especificidades 
da lesão e do paciente, indicação de cobertura, tipo de curativo a serem utilizados no controle dessas lesões. Provavelmente, essas limitações estão relacionadas à ausência de educação permanente no serviço de saúde e educação continuada em áreas afins, tais como: Dermatologia, Estomaterapia, Cuidados Paliativos dentre outros.

Outro aspecto preocupante é a omissão da assistência de enfermagem direcionada a estes pacientes. Acreditase que a falha na realização da avaliação e tratamento de pacientes com feridas neoplásicas esteja relacionada a alguns fatores, dentre eles: déficit do conhecimento relacionado aos conteúdos e técnicas no cuidado às feridas neoplásicas; aumento da carga de trabalho devido às atividades gerenciais; mal planejamento do dimensionamento de pessoal, de insumos e de materiais para a avaliação de feridas, assim como produtos, substâncias e coberturas escassos no serviço de saúde.

Nesse sentido, os resultados desse estudo assinalam a necessidade educação permanente no serviço de saúde, locus da pesquisa, a fim de treinar a equipe de enfermagem para o acompanhamento de pacientes com lesões neoplásicas, bem como estruturação de unidade de cuidados paliativos, com recursos humanos e materiais necessários, criação e implantação de protocolos assistenciais que norteiem a prática de métodos avaliativos e terapêuticos no cuidado a pessoas com feridas neoplásicas, familiares e cuidadores.

Este estudo apresenta limitações quanto à amostra,uma vez que foi pequena e apenas enfermeiros de uma mesma instituição foram pesquisados. Por esse motivo, o estudo precisa ser replicado, após refinamento do instrumento, com amostra maior e em outras instituições de saúde, para que se tenha um panorama de como o conhecimento e a prática do cuidado a pacientes com feridas neoplásicas está sendo realizado.

Outra limitação do estudo está relacionada à discussão dos resultados, no que refere-se à escassez de dados comparativos com outras pesquisas. Neste sentido, vale ressaltar que os estudos publicados em âmbito nacional e internacional acerca da temática apresentam, geralmente, delineamento bibliográfico, o que contribui para níveis de evidência baixos, inviabilizando a comparação, considerada condição sine qua non para confirmar ou refutar as hipóteses da pesquisa.

Embora se reconheça as limitações do estudo, considera-se fundamental tomar os resultados como forma de reflexão sobre a importância da educação em saúde no processo de formação de enfermeiros. Para tanto, a educação necessita ser também integral e interdisciplinar, com bases em referenciais críticos-reflexivos, permitindo a aquisição de competências e habilidades que garantam ações voltadas para a pessoa com ferida neoplásica em sua subjetividade.

\section{REFERÊNCIAS}

1. Guimarães RM, Muzi CD, Teixeira MP, Pinheiro SS. A transição da mortalidade por cânceres no Brasil e a tomada de decisões estratégicas nas políticas públicas de saúde da mulher. Rev Pol Pub. 2016; 20(1):33-50.

2. Maida V, Alexander S, Case AA, Fakhraei P. Malignant wound management. Public Health Emerg. 2016. Jun; 1:12.

3. Instituto Nacional de Câncer. Brasil. Estimativa 2016/2017: Incidência de Câncer no Brasil. Rio de Janeiro: INCA, 2016.

4. illey C, Lipson J, Ramos M. Palliative wound care for malignant fungating wounds: holistic considerations at end-of-life. Nurs Clin N Am. 2016; 51(3):513-31.

5. Beh SY, Leow LC. Fungating breast cancer and other malignant wounds: epidemiology, assessment and management. Expert Rev Qual Life Cancer Care. 2016. Mar; 1(2):137-44.

6. Gozzo TO, Tahan FP, Andrade M, Nascimento TG, Prado MAS. Ocorrência e manejo de feridas neoplásicas em mulheres com câncer de mama avançado. Esc Anna Nery. 2014; 18(2):270-6.

7. Lisboa IND, Valença MP. Caracterização de Pacientes com Feridas Neoplásicas. Estima 2016; 14(1):21-8.

8. Agra G, Ferreira TMC, Oliveira DMN, Nogueira WP, Brito DTF, Oliveira OS et al. Neoplastic wounds: controlling pain, exudate, odor and bleending. Int Arch Med. 2017.10(118):1-11.

9. Agra G, Santos JP, Sousa ATO, Gouveia BLA, Brito, DTF, Macêdo EL et al. Malignant neoplastic wounds: clinical management performed by nurses. Int Arch Med. 2016; 9(344):1-13.

10. Instituto Nacional de Câncer. Brasil. Tratamento e controle de feridas tumorais e úlceras por pressão no câncer avançado. Rio de Janeiro: INCA, 2011.

11. Harris DG, Noble SIR. Management of terminal hemorrhage in patients with advanced cancer: a systematic literature review. J Pain Symptom Manage. 2009 Dec; 38(6):913-27.

12. Fromantin I, Watson S, Baffie A, Rivat A, Falcou MC, Kriegel I, Ingenior YR. A prospective, descriptive cohort study of malignant wound characteristics and woound care strategies in patients with breast cancer. Ostomy Wound Mange. 2014. Jun; 60(6):38-48.

13. Dealey C. Cuidando de feridas: um guia para enfermeiras. $3^{a}$ ed. São Paulo: Ateneu, 2008.

14. National Pressure Ulcer Advisory Panel (NPUAP), European Pressure Ulcer Advisory Panel (EPUAP) and Pan Pacific Pressure Injury Alliance (PPPIA). Prevention and Treatment of Pressure Ulcers: Quick Reference Guide. Emily Haesler (Ed.). Cambridge Media: Perth, Australia; 2014.

15. Santos CMC, pimenta CAM, Nobre MRC. A systematic review of topical treatments to control the odor of malignant fungating wounds. J Pain Symptom Manage. 2010. 39(6):1065-76.

16. Oliveira RAO, Nigri EL. Sutura elástica no tratamento de escarotomias e fasciotomias de pacientes queimados. Rev Bras Queimaduras. 2012;11(2):63-6.

17. Martins EAP, Meneghin P.Avaliação de três técnicas de limpeza do sítio cirúrgico infectado utilizando soro fisiológico. Cienc Cuid Saude. 2012; 11:204-10.

18. Lund-Nielsen B, Muller K, Adamsen L. Qualitative and quantitative evaluation of a new regimen for malignant wounds in women with advanced breast cancer.J Wound Care. 2005 Feb; 14(2):69-73. 
19. Segovia D. The clinical benefits of Active Leptospermum honey in oncologic wounds. Ostomy W Manage. 2010 Oct; 56(10):14-17.

20. Seaman S, Bates-Jensen BM. Skin disorders. Malignant wounds, fistulas, and stomas. In: Ferrell BR, Coyle N, Paice JA. Oxford Textbok of palliative nursing. $4^{a}$ ed. Oxford University Press, 2015, p.325-30.

21. Santos WA, Fuly PSC, Santos MLSC, Souto MD, Reis CM, Beretta LL. Evidências sobre o isolamento social em pacientes com exsudato em feridas neoplásicas: revisão integrativa. Revi Enferm Atual. 2017; 81(17)- 81-8. 